\title{
Targeting double genes in multiplex PCR for discriminating bovine, buffalo and porcine materials in food chain
}

\begin{abstract}
Beef, buffalo and pork are the major meat of economic, religious and health concern. Current methods to authenticate these materials in food chain are based on mainly single gene targets which are susceptible to break down by food processing treatments. We, for the first time, described here a double gene targeting short-amplicon length multiplex polymerase chain reaction assay for discriminating bovine, buffalo and porcine materials in a single assay platform. The advantage of the assay is evidenced in terms of fidelity, cost and time since it is highly unlikely that two different targets would be missing even in a decomposed specimen. Detection of multiple targets in a single assay definitely saves analytical cost and time. Mitochondrial cytochrome b (cytb) and ND5 genes were targeted and six different targets (length: 90-146 bp), two for each of cow (120 and 106bp), buffalo (90 and 138bp) and pig (73 and 146bp), were amplified from raw, boiled, autoclaved and microwaved cooked meat under pure and mixed matrices. The detection limit was $0.02 \mathrm{ng}$ DNA under pure states and $0.1 \%$ meat in binary mixtures and meatball products. Screening of Malaysian meatball products revealed all beef products were buffalo positive in which $35 \%$ were totally replaced. In contrast, all pork products were found uncontaminated from beef and buffalo.
\end{abstract}

Keyword: Multiplex PCR; Double gene-targets; Short-length DNA targets; Economic; Religious and health concern; Decomposed specimens; Meatball products 